Version of Record: https://www.sciencedirect.com/science/article/pii/S0196655318309167

Manuscript_3333aebe71dfcf6e3bda38591d1e72d8

Major Article

\title{
Impact of an Acinetobacter baumannii outbreak on kidney events in a burn unit: A targeted machine learning analysis
}

\author{
Thomas Vauchel MD ${ }^{\mathrm{a}}$, Romain Pirracchio MD, PhD ${ }^{\mathrm{b}, \mathrm{c}}$, Maïté Chaussard MD ${ }^{\mathrm{a}, \mathrm{d}}$, Matthieu Lafaurie MD ${ }^{\mathrm{e}}$, \\ Martine Rouveau MD ${ }^{\mathrm{f}}$, Clotilde Rousseau MD ${ }^{\mathrm{f}}$, Mourad Benyamina MD ${ }^{\mathrm{a}}$, Sabri Soussi MD ${ }^{\mathrm{a}}$, \\ François Dépret MD ${ }^{\mathrm{a}, \mathrm{d}}$, Axelle Ferry MD ${ }^{\mathrm{a}}$, Haikel Oueslati MD ${ }^{\mathrm{a}}$, Marc Chaouat MD, PhD ${ }^{\mathrm{d}, \mathrm{g}}$, \\ Maurice Mimoun MD, PhD ${ }^{\mathrm{d}, \mathrm{g}}$, Vincent Jarlier MD ${ }^{\mathrm{h}}$, Nabila Moreno MD ${ }^{\mathrm{i}}$, Alexandre Mebazaa MD, PhD ${ }^{\mathrm{a}, \mathrm{d}, \mathrm{j}, \mathrm{k}}$, \\ Matthieu Legrand MD, PhD ${ }^{\mathrm{a}, \mathrm{d}, \mathrm{j}, \mathrm{k}, *}$
}

a AP-HP, GH St-Louis-Lariboisière, Department of Anesthesiology and Critical Care and Burn Unit, Paris, France

${ }^{\mathrm{b}}$ AP-HP, Hôpital Européen Georges Pompidou, Service d'Anesthesie-Reanimation, Paris, France

' Service de Biostatistique et Informatique Médicale, INSERM UMR-1153, Equipe ECSTRA, Hôpital Saint-Louis, Sorbonne Paris Cité, Paris, France

${ }^{\mathrm{d}}$ Université Paris Diderot, Paris, France

e AP-HP, GH St-Louis-Lariboisière, Service de Maladies Infectieuses, Paris, France

${ }^{\mathrm{f}}$ AP-HP, GH St-Louis-Lariboisière, Service de Bactériologie, Paris, France

${ }^{g}$ AP-HP, GH St-Louis-Lariboisière, Plastic Surgery and Burn Unit, Paris, France

${ }^{\mathrm{h}}$ AP-HP, Hôpital La Pitié-Salpêtrière, Laboratoire de Bactériologie-Hygiène, Paris, France

${ }^{\mathrm{i}}$ Service de biochimie, Hôpital Saint-Louis, Paris, France

JUR INSERM 942, Institut National de la Santé et de la Recherche Médicale (INSERM), Paris, France

${ }^{\mathrm{k}}$ F-CRIN, INICRCT Network, Paris, France.

Key Words:

Renal failure

Outcome

Nephrotoxic

Multidrug-resistant bacteria

Intensive care

\begin{abstract}
Background: Multidrug-resistant (MDR) bacteria outbreaks represent a major threat in intensive care units. Patients may then be exposed to drug-related direct toxicity during such outbreaks. The objective of this study was to explore the impact of an outbreak of imipenem-resistant Acinetobacter baumannii (IR-AB) on renal outcomes. Methods: We performed a before-and-after observational study in a French burn intensive care unit during an IR-AB outbreak: a 13-month period before (period A, October 2013-October 2014) and a 13-month period after outbreak control (period B, December 2014-December 2015). A total of 409 patients were included, 195 during period $A$ and 214 during period $B$. The main endpoint was major adverse kidney events at day 90 (MAKE 90). Secondary endpoints were acute kidney injury (AKI) and persistent renal dysfunction.

Results: Incidence of MAKE 90 was $15.9 \%$ during period A versus $11.2 \%$ during period $B(P=.166)$ and AKI $28.2 \%$ versus $18.7 \%(P=.023)$. The use of colistin was associated with renal outcomes in univariate analysis. After adjustment of potential confounding factors using a targeted Machine Learning Analysis (ie, IR-AB-related infection, septic shock, severity scores, other nephrotoxics, chronic kidney disease, serum creatinine at admission, Staphylococcus aureus), colistin remained associated with the risk of MAKE and AKI (relative risk $=2.909,95 \%$ confidence interval $[\mathrm{Cl}][1.364$, $6.204], P=.006$ for MAKE 90 , and relative risk $=2.14,95 \% \mathrm{CI}[1.52,3.02], P<.0001$ for AKI).
\end{abstract}

Conclusions: The episode of IR-AB outbreak was associated with an increased risk of kidney events, which appears to be driven by the use of colistin.

(C) 2018 Association for Professionals in Infection Control and Epidemiology, Inc. Published by Elsevier Inc. All rights reserved.

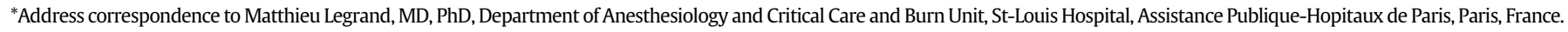
E-mail address: matthieu.legrand@aphp.fr (M. Legrand).

Conflicts of interest: Dr. Legrand reports grants and personal fees from Sphingotec, personal fees from Novartis, personal fees from Fresenius, grants from French Ministry of Health, all outside the submitted work.

Authors contributions: T.V., M.C., M.L., M.R., C.R., M.B., S.S., F.D., A.F., H.O., M.C., M.M., V.J, N.M., A.M., and M.L. collected the data and modified the drafted version. A.M. and M.M.

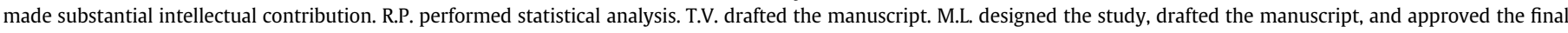
version. All authors approved the final version.

Ethics approval and consent to participate: This study was approved by our local ethical committee (IRB 00003835, protocol 2013/17NICB) and was in accordance to the Helsinki declaration. Consent to participate was waived.

Availability of data and materials: The dataset supporting the conclusions in this article will be made available from the corresponding author upon request.
} 


\section{BACKGROUND}

The emergence of multidrug-resistant (MDR) bacteria represents a global threat around the world. Intensive care units (ICUs) are at risk of MDR bacteria outbreaks. Among MDR bacteria, Acinetobacter baumannii $(\mathrm{AB})$ is a nonfermentative gram-negative bacillus that may be isolated from soil and water, as well as from human skin, and gastrointestinal and upper respiratory tracts. The resistance of $A B$ to desiccation over extended periods is responsible for a high rate of cross-transmission. $\mathrm{AB}$ has been involved in healthcare-associated infections especially in immunocompromised patients ${ }^{1,2}$ and carries with it various forms of resistance. ${ }^{3}$

Previous studies have reported that MDR bacteria infections are associated with poor outcomes. ${ }^{4-7}$ This association could result from the virulence of the bacteria itself or inadequate initial antibiotic treatment. A third reason could be the toxicity of antibiotics used to treat MDR bacteria, such as colistin. Colistin belongs to the family of polymyxins and is often the last antibiotic available to treat such severe MDR bacteria. Colistin has been associated with renal toxicity. In turn, acute kidney injury (AKI) is known to be associated with poor outcomes in hospitalized patients and especially in critically ill burn patients (ie, total burn surface area greater than $20 \%$ or patients with organ failure). We hypothesize that an imipenem-resistant Acinetobacter baumannii (IR-AB) outbreak in a unit might globally increase the exposure to colistin and thus increase the incidence of adverse renal events. In the present study, we evaluated the direct impact of an IR-AB outbreak on major kidney events through colistin use in critically ill burn patients.

\section{METHODS}

This study was performed from a prospective database in a French burn ICU of a tertiary teaching hospital. This study was approved by our local ethical committee (IRB 00003835, protocol 2013/17NICB) and was in accordance with the Helsinki declaration.

\section{Patients}

All consecutive patients older than 18 years admitted in the unit during 2 specific periods were included in the study. Exclusion criteria were end-stage renal disease, renal replacement therapy prior to admission, and burn unit length of stay $<24$ hours. The following information was collected: demographic characteristics (age, sex, height, weight, comorbidities and previous treatments including $\beta$-blockers, angiotensin-converting enzyme inhibitors, diuretics, treatment of diabetes, statins), admission characteristics (total burn surface area), mechanism of burn injury, smoke inhalation (defined as exposition to smoke with symptoms of smoke inhalation), ${ }^{8}$ Simplified Acute Physiology Score II, ${ }^{9}$ Abbreviated Burn Severity Index, ${ }^{10}$ infection characteristics (source of infection and pathogens), renal outcome, organ supports, and 90-day mortality.

The study period therefore included a first 13-month period during an IR-AB outbreak in the burn unit of Saint-Louis hospital in Paris (period A, between October 2013 and October 2014) and a second 13-month period with no IR-AB colonization or infection episodes (period B, between December 2014 and December 2015). The unit closed for 1 month in November 2014 between the 2 periods for complete surface cleaning and outbreak control.

The comparison of the 2 periods allowed us to evaluate the impact of the IR-AB outbreak and colistin exposure from other factors and treatments on renal outcomes in patients admitted to our burn unit.

\section{Empirical antimicrobial treatment}

The decision to initiate antibiotics was made after an infection was diagnosed or highly suspected. Except in patients with a known or reported allergy, probabilistic antibiotics included a $\beta$-lactam (ie, broad-spectrum cephalosporins, carbapenems, or ureidopenicillins with $\beta$-lactamase inhibitors). The decision to use colistin and/or aminoglycosides was based on the presence of a known colonization with bacteria resistant to $\beta$-lactam and/or the occurrence of severe sepsis or septic shock before any microbiological documentation while other patients hospitalized in the unit were colonized or infected with MDR bacteria (susceptible to aminoglycosides or colistin). Colistin was administrated intravenously with a loading dose of 9 million international units (MUI) and maintenance dose of 4.5 MUI twice a day. The colistin dose was secondly adjusted based on glomerular filtration rate. Furthermore, vancomycin was prescribed in case of a known colonization with methicillin-resistant Staphylococcus aureus or the occurrence of severe sepsis or septic shock before any microbiological documentation while other patients hospitalized in the unit were colonized or infected with methicillin-resistant Staphylococcus aureus.

Treatment modifications (ie, escalation, de-escalation, or interruption) were discussed on a daily basis during rounds. Furthermore, modifications and definitive diagnostics of infections were validated twice a week during multidisciplinary medical meetings, including infectious disease specialists, intensivists, surgeons, and microbiologists.

\section{Study endpoints}

The primary endpoint measure was the occurrence of a major adverse kidney event within the first 90 days following ICU admission (MAKE $90^{11}$ ), defined as at least 1 of the following events: need for renal replacement therapy, persistent renal dysfunction (discharge creatinine $>120 \%$ admission creatinine), and/or death. Secondary endpoints were AKI (defined by the Kidney Disease - Improving Global Outcome- KDIGO classification ${ }^{12}$ ), severe AKI (defined as stage 2 or $3 \mathrm{AKI}$ ), and persistent renal dysfunction.

\section{Statistical analysis}

Continuous variables are described using median (first-third quartiles). Categorical variables are described using count (\%). Categorical variables were compared using the Fisher test, continuous variables using the Wilcoxon test.

The association between colistin exposure and MAKE 90 was assessed by estimating the average treatment effect using targeted maximum likelihood estimation (TMLE). ${ }^{13,14}$ This estimation was adjusted on the following variables: Simplified Acute Physiology Score II; Sequential Organ Failure Assessment score at admission; Abbreviated Burn Severity Index; use of vancomycin, aminoglycosides, amphotericin B, or intravenous contrast agent during the first 90 days; presence of septic shock; history of chronic kidney disease; or creatinine at admission. A confirmed infection related to IR-AB or $S$ aureus was integrated to the model. The results are expressed as relative risks, together with their 95\% confidence intervals (CIs), and the corresponding $P$ values.

TMLE is a 2-step procedure. The first step consists of modeling the expected value of the outcome given the treatment of interest (ie, colistin) and all relevant covariates. In our case, this first step involved super learning, a hybrid machine learning meta-algorithm that can leverage the best from a library of several possible parametric and nonparametric algorithms using cross-validation. ${ }^{15}$ In a second step, the TMLE procedure updates the initial estimate 
using a fit of the propensity score, in order to obtain an optimal bias-variance tradeoff for the parameter of interest. Again, in our case, the propensity score was fitted using super learning. TMLE was previously shown to be double robust. ${ }^{16}$ However, while doubly robust estimators facilitate inference when all relevant regression functions (ie, the expected value of the outcome given the treatment of interest and the propensity score) are consistently estimated, the same cannot be said when at least 1 estimator is inconsistent. To overcome this limitation, we used a novel version of the TMLE, called double-robust TMLE, that implements doubly robust CIs and allows for accurate inference even when the regression functions are not consistently estimated.

All analyses were performed using R 2.15.1 statistical software running on a Mac OsX platform (SuperLearner and drtmle packages, The R Foundation for Statistical Computing, Vienna, Austria).

\section{RESULTS}

\section{Characteristics of patients}

A total of 436 patients were admitted to the ICU during the 2 study periods. Eight patients were excluded because of treatment with renal replacement therapy before an episode of infection, 9 patients were excluded because of an ICU stay less than 24 hours, and 10 patients were not included because of missing data. Finally, 409 patients were included in the study, 195 during period A and 214 during period B. Patients' characteristics are summarized in Table 1.

\section{Renal outcome}

The incidence of MAKE 90 was not statistically different between the 2 periods ( $15.9 \%$ vs $11.2 \%, P=.166)$. At day 90 , there was no difference in mortality ( $13.3 \%$ vs $7.5 \%, P=.051)$ but more episodes of AKI (28.2\% vs $18.7 \%, P=.023)$ and more persistent renal dysfunction (13.3\% vs 7.0\%, $P=.033$ ) during period A (Fig 1 ).

\section{Impact of IR-AB and colistin use on outcomes}

Fifty-six patients (29\%) were colonized with IR-AB during period A versus $0 \%$ during period $\mathrm{B}(P<.001)$. Of note, more patients had infections due to methicillin-resistant $S$ aureus during period $A$ than during period $B$ (Table 2 ). Twenty-one patients had a confirmed infection with IR-AB (11\%), and $33(16.9 \%)$ patients received colistin during period A versus $0 \%$ during period $\mathrm{B}(P<.001)$, as well as more aminoglycoside $(17.4 \%$ vs $4.2 \%, P<.001)$, more vancomycin $(9.2 \%$ vs $0.9 \%$, $P<.001)$, and more iodinated contrast media (10.8\% vs $3.3 \%, P=.003$ ). The use of liposomal amphotericin B did not differ between the 2 periods ( $1.5 \%$ vs $3.7 \%, P=.170)$.

After adjustment of potential confounding factors, the use of colistin was independently associated with the risk of MAKE and AKI (relative risk $(\mathrm{RR})=2.9,95 \% \mathrm{CI}[1.36-6.20], P=.006$ for MAKE at day 90, and $\mathrm{RR}=2.14,95 \% \mathrm{CI}[1.52,3.02], P<.0001$ for AKI at day 90 ). Colistin use was not associated with mortality $(\mathrm{RR}=1.85,95 \% \mathrm{CI}$ $[0.34,9.96], P=.473$ ) or persistent renal dysfunction $(\mathrm{RR}=0.80,95 \%$ CI $[0.03,23.46], P=.896)$. Of note, IR-AB-related infections weren't associated with an increased adjusted risk of MAKE $90(\mathrm{RR}=2.11,95 \%$ CI [0.76 5.86], $P=.15)$.

\section{DISCUSSION}

In this cohort, the use of colistin was associated with the risk of MAKE and AKI after adjustment for severity score and infections due to IR-AB. These results strongly suggest that IR-AB outbreaks might impact renal outcome through the use of colistin. This association implies that patients hospitalized during an IR-AB
Table 1

Characteristics of patients at admission

\begin{tabular}{|c|c|c|c|c|}
\hline $\begin{array}{l}\text { Patients } \\
\text { characteristics }\end{array}$ & $\begin{array}{l}\text { All patients } \\
(\mathrm{N}=409)\end{array}$ & $\begin{array}{l}\text { Period A } \\
(\mathrm{N}=195)\end{array}$ & $\begin{array}{l}\text { Period B } \\
(\mathrm{N}=214)\end{array}$ & $P$ value \\
\hline \multicolumn{5}{|l|}{ Demographic data } \\
\hline Age & $47.0(28)$ & $49.0(19)$ & $46.0(20)$ & .408 \\
\hline Women & $168(41.1 \%)$ & 77 (39.5\%) & $91(42.5 \%)$ & .533 \\
\hline Men & $241(58.9 \%)$ & $118(60.5 \%)$ & $123(57.5 \%)$ & .533 \\
\hline BMI & $24(6)$ & $24(6.4)$ & $24.5(6.0)$ & .658 \\
\hline \multicolumn{5}{|l|}{ Comorbidities } \\
\hline COPD & $8(2.0 \%)$ & $7(3.6 \%)$ & $1(0.5 \%)$ & .023 \\
\hline Diabetes & $53(13.0 \%)$ & $24(12.3 \%)$ & $29(13.6 \%)$ & .708 \\
\hline HBP & $95(23.2 \%)$ & $50(25.6 \%)$ & $45(21 \%)$ & .270 \\
\hline Chronic heart failure & $10(2.4 \%)$ & $7(3.6 \%)$ & $3(1.4 \%)$ & .152 \\
\hline Chronic renal failure & $9(2.2 \%)$ & $0(0 \%)$ & $9(4.2 \%)$ & .004 \\
\hline Stroke & $11(2.7 \%)$ & $7(3.6 \%)$ & $4(1.9 \%)$ & .283 \\
\hline Immunosuppression & $8(2.0 \%)$ & $4(2.1 \%)$ & $4(1.9 \%)$ & .894 \\
\hline \multicolumn{5}{|l|}{ Burn characteristics } \\
\hline Thermal & $346(90.6 \%)$ & $157(89.7 \%)$ & $189(91.3 \%)$ & \\
\hline Electric & $26(6.8 \%)$ & $14(8.0 \%)$ & $12(5.8 \%)$ & .839 \\
\hline Chemical & $10(2.6 \%)$ & $4(2.3 \%)$ & $6(2.9 \%)$ & \\
\hline Hydroxocobalamin & $21(5.5 \%)$ & $12(6.8 \%)$ & $9(4.4 \%)$ & .295 \\
\hline TBSA (\%) & $11(19)$ & $14(18.5)$ & $10(16.6)$ & .146 \\
\hline \multicolumn{5}{|l|}{ Severity scores } \\
\hline ABSI & $6(4)$ & $6(3)$ & $6(3)$ & .151 \\
\hline SAPSII & $19(16)$ & $19(17)$ & $19(16)$ & .718 \\
\hline SOFA & $0(2)$ & $0(2.24)$ & $0(2.20)$ & .023 \\
\hline \multicolumn{5}{|l|}{ Other parameters } \\
\hline Catecholamine & $52(12.7 \%)$ & $28(14.4 \%)$ & $24(11.2 \%)$ & .34 \\
\hline Creatinine & $71(31)$ & $73(70)$ & $68(36)$ & .184 \\
\hline
\end{tabular}

NOTE: Continuous variables: Median (IQR); Nominal variables: Number (\%). $A B S I$, abbreviated burn severity index; $B M I$, body mass index; $C O P D$, chronic obstructive pulmonary disease; $H B P$, high blood pressure; $M A K E$, major adverse kidney event; SAPSI, simplified acute physiology score; SOFA, sequential organ failure assessment; TBSA, total burned surface area.

outbreak might be exposed to drug-related adverse events due to the large use of colistin.

The impact on mortality and morbidity of MDR bacteria-related infection has been debated. A meta-analysis published in 2006 reported an increased risk of death in patients infected with $A B .{ }^{17}$ In a more recent meta-analysis, Falagas et al reported an increased risk of dying after infections due to carbapenem-resistant Enterobacteriae. ${ }^{18}$ In a recent retrospective study, bacteremia to carbapenem-resistant gram-negative bacteria was associated with an increase length of stay and mortality. ${ }^{19}$ More recently, multidrug-resistant infections were estimated to account

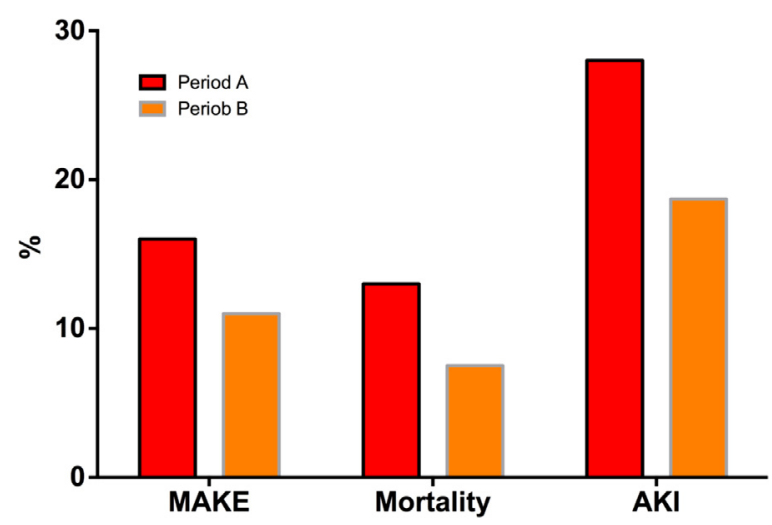

Fig 1. Incidence of events between the 2 periods. The study period included a first 1 -year period during the imipenem-resistant Acinetobacter baumannii (IR-AB) outbreak (Period A, between October 2013 and October 2014) and a second 1-year period after the unit closed in November 2014 for complete surface cleaning and control of the outbreak (Period B, between December 2014 and December 2015). MAKE, major associated kidney events at day 90; AKI, acute kidney injury at day 90. 
Table 2

Characteristics of infections

\begin{tabular}{lcccc}
\hline Infections characteristics & All patients & Period A & Period B & P value \\
\hline Source of infections & & & & \\
Cutaneous & $69(16.9 \%)$ & $39(20 \%)$ & $30(14 \%)$ & .107 \\
Pulmonary & $79(19.3 \%)$ & $50(25.6 \%)$ & $29(13.6 \%)$ & .002 \\
Abdominal & $7(1.7 \%)$ & $5(2.6 \%)$ & $2(0.9 \%)$ & .204 \\
Urinary & $6(1.5 \%)$ & $4(2.1 \%)$ & $2(0.9 \%)$ & .348 \\
Catheter & $13(3.2 \%)$ & $8(4.1 \%)$ & $5(2.3 \%)$ & .309 \\
ENT & $1(0.2 \%)$ & $0(0 \%)$ & $1(0.5 \%)$ & .339 \\
Unknown & $15(3.7 \%)$ & $11(5.6 \%)$ & $4(1.9 \%)$ & .043 \\
Bacteraemia & $70(17.1 \%)$ & $40(20.5 \%)$ & $30(14 \%)$ & .082 \\
Pathogen & & & & \\
Acinetobacter baumannii & $21(5.1 \%)$ & $21(10.8 \%)$ & $0(0 \%)$ & $<.0001$ \\
Pseudomonas aeruginosa & $39(9.5 \%)$ & $23(11.8 \%)$ & $16(7.5 \%)$ & .138 \\
Enterobacterieae & $78(19.1 \%)$ & $45(23.1 \%)$ & $33(15.4 \%)$ & .049 \\
Other GNB & $18(4.4 \%)$ & $9(4.6 \%)$ & $9(4.2 \%)$ & .840 \\
Staphylococcus aureus & $42(10.3 \%)$ & $25(12.8 \%)$ & $17(7.9 \%)$ & .105 \\
MRSA & $11(2.7 \%)$ & $9(4.6 \%)$ & $2(0.9 \%)$ & .022 \\
Other GPC & $45(11 \%)$ & $29(14.9 \%)$ & $16(7.5 \%)$ & .017 \\
Aspergillus & $3(0.7 \%)$ & $1(0.5 \%)$ & $2(0.9 \%)$ & .618 \\
Mucor & $7(1.7 \%)$ & $3(1.5 \%)$ & $4(1.9 \%)$ & .797 \\
Other fungal germ & $13(3.2 \%)$ & $7(3.6 \%)$ & $6(2.8 \%)$ & .651 \\
\hline
\end{tabular}

ENT, ear nose throat; GNB, gram-negative bacilli; GPC, gram-positive cocci; MRSA, methicillin-resistant $\mathrm{S}$ aureus.

for $12 \%$ of the overall mortality of healthcare-associated infections, as well as an increase in the hospital readmission rate. ${ }^{20}$ Inappropriate probabilistic antibiotics can jeopardize the chance of recovery and negatively impact outcomes. When facing a patient with signs of sepsis or septic shock, physicians are keen to treat the patient's colonization and MDR pathogens of other patients hospitalized in this ICU with initial broadspectrum antibiotics, including colistin, during MDR bacteria outbreaks. We hypothesize that the potential toxic effects of colistin on the kidney may also be involved in the negative impact of MDR bacteria on outcomes. Colistin belongs to the family of polymyxins, polymyxins E, discovered in the 1950s. It acts by modifying lipopolysaccharide, increasing the permeability of the bacterial membrane, and leading to cell lysis. Its elimination is mainly renal in unchanged form (about 70\%); the mechanisms of action of the elimination of its derivatives are currently unclear. ${ }^{21}$

Accumulating evidence suggests that occurrence of AKI leads to remote organ injury and poor outcomes in critically ill patients. In the present study, the use of colistin was associated with a risk of AKI and major adverse events. The results of our study provide additional evidence that efforts should target the prevention of MDR bacteria spreading as well as the rapid identification of bacteria phenotypes and genotypes in severely ill infected patients to prevent such renal adverse events. Rapid identification of MDR bacteria could allow tailored antibiotic treatments and restrictive use of toxic antibiotics such as colistin. Implementation of such a strategy may prove to limit the risk of AKI and improve global outcomes.

Our study suffers from several limitations. First, it was a singlecenter study, which limits the generalizability of the results. Second, the design does not allow drawing any definitive conclusions regarding the impact of the outbreak on renal outcomes. However both the pathophysiological background of kidney toxicity of colistin and the robust statistical analysis, using machine learning, strongly suggest that such a causal relationship exists. Also, our protocols were not modified between the 2 periods. Finally, performing a randomized controlled trial in this setting is not feasible. Of note, the IR-AB outbreak was associated with a global increase in the use of nephrotoxic drugs, including aminoglycosides, vancomycin, and contrast media, which underlines the challenge to treat infections leading to combined antibiotics treatments and more frequent imaging. However, after adjustment for all nephrotoxic agents, colistin remained associated with poor renal outcome, suggesting the high burden on this specific drug.

\section{CONCLUSIONS}

To conclude, the use of colistin during an IR-AB outbreak was associated with an increased risk of renal events. Such observations suggest that preventing IR-AB outbreaks may improve patients' renal outcomes. Rapid microbiological susceptibility testing (eg, using molecular diagnostic methods) could also prevent the empirical use of nephrotoxic drugs.

\section{Acknowledgment}

The authors thank the care providers of the burn unit of Saint-Louis Hospital.

\section{References}

1. Schreckenberger PC, Daneshvar MI, Weyant RS, Hollis DG. Acinetobacter, Achromobacter, Chryseobacterium, Moraxella, and other nonfermentative Gramnegative rods. In: Murray PR, Baron EJ, Jorgensen JH, Landry ML, Pfaller MA, eds. Man Clin Microbiol 9th Ed Wash DC ASM Press. 2007;770-802.

2. Shelby J, Merrell SW. In vivo monitoring of postburn immune response. J Trauma 1987;27:213-6.

3. Munoz-Price LS, Weinstein RA. Acinetobacter infection. N Engl J Med 2008;358: 1271-81.

4. Tabah A, Koulenti D, Laupland K, Misset B, Valles J, Bruzzi de Carvalho F, et al. Characteristics and determinants of outcome of hospital-acquired bloodstream infections in intensive care units: the EUROBACT International Cohort Study. Intensive Care Med 2012;38:1930-45.

5. Leão ACQ Menezes PR, Oliveira MS, Levin AS. Acinetobacter spp. are associated with a higher mortality in intensive care patients with bacteremia: a survival analysis. BMC Infect Dis 2016;16:386.

6. Wareham DW, Bean DC, Khanna P, Hennessy EM, Krahe D, Ely A, et al. Bloodstream infection due to Acinetobacter spp: epidemiology, risk factors and impact of multi-drug resistance. Eur J Clin Microbiol Infect Dis 2008;27:607-12.

7. Falagas ME, Rafailidis PI. Attributable mortality of Acinetobacter baumannii: no longer a controversial issue. Crit Care 2007;11:134.

8. Sheridan RL. Fire-related inhalation injury. N Engl J Med 2016;375:1905.

9. Le Gall JR, Lemeshow S, Saulnier F. A new Simplified Acute Physiology Score (SAPS II) based on a European/North American multicenter study. JAMA 1993;270: 2957-63.

10. Tobiasen J, Hiebert JM, Edlich RF. The abbreviated burn severity index. Ann Emerg Med 1982;11:260-2.

11. Billings IV FT, Shaw AD. Clinical trial endpoints in acute kidney injury. Nephron Clin Pract 2014 Sep;127:89-93

12. Kidney Disease. Improving Global Outcome (KDIGO) Acute Kidney Injury Work Group. KDIGO clinical practice guideline for acute kidney injury. Kidney Int Suppl 2012; (Supp 2):1-138.

13. Zheng W, van der Laan MJ. Cross-validated targeted minimum-loss-based estimation. In: Targeted learning. Springer, New York (NY); 2011 [cited 2017 Nov 4]. p. 459-74. (Springer Series in Statistics). Available from: https://link.springer.com/ chapter/10.1007/978-1-4419-9782-1_27. Accessed November 12, 2018.

14. Legrand M, Pirracchio R, Rosa A, Petersen ML, Van der Laan M, Fabiani J-N, et al. Incidence, risk factors and prediction of post-operative acute kidney injury following cardiac surgery for active infective endocarditis: an observational study. Crit Care Lond Engl 2013;17:R220.

15. van der Laan MJ, Polley EC, Hubbard AE. Super learner. Stat Appl Genet Mol Biol 2007;6: Article25.

16. Zheng W, Petersen M, van der Laan MJ. Doubly robust and efficient estimation of marginal structural models for the hazard function. Int J Biostat 2016;12:233-52.

17. Falagas ME, Bliziotis IA, Siempos II. Attributable mortality of Acinetobacter baumannii infections in critically ill patients: a systematic review of matched cohort and case-control studies. Crit Care Lond Engl 2006;10:R48.

18. Falagas ME, Tansarli GS, Karageorgopoulos DE, Vardakas KZ. Deaths attributable to carbapenem-resistant Enterobacteriaceae infections. Emerg Infect Dis 2014 Jul;20: 1170-5.

19. Ting S-W, Lee C-H, Liu J-W. Risk factors and outcomes for the acquisition of carbapenem-resistant Gram-negative bacillus bacteremia: a retrospective propensity-matched case control study. J Microbiol Immunol Infect. 2018;51:621-8.

20. Barrasa-Villar JI, Aibar-Remón C, Prieto-Andrés P, Mareca-Doñate R, MolinerLahoz J. Impact on morbidity, mortality, and length of stay of hospital-acquired infections by resistant microorganisms. Clin Infect Dis 2017;65:644-52.

21. Dudhani RV, Turnidge JD, Nation RL, Li J. fAUC/MIC is the most predictive pharmacokinetic/pharmacodynamic index of colistin against Acinetobacter baumannii in murine thigh and lung infection models. J Antimicrob Chemother 2010; 65:1984-90. 